\title{
Self-assembly of Janus particles into helices with tunable pitch
}

\author{
M. Sobrino Fernández, ${ }^{*}$ V. R. Misko, ${ }^{\dagger}$ and F. M. Peeters ${ }^{\ddagger}$ \\ Departement Fysica, Universiteit Antwerpen, B-2020 Antwerpen, Belgium \\ (Received 18 March 2015; revised manuscript received 16 June 2015; published 15 October 2015)
}

\begin{abstract}
Janus particles present an important class of building blocks for directional assembly. These are compartmentalized colloids with two different hemispheres. In this work we consider a three-dimensional model of Janus spheres that contain one hydrophobic and one charged hemisphere. Using molecular dynamics simulations, we study the morphology of these particles when confined in a channel-like environment. The interplay between the attractive and repulsive forces on each particle gives rise to a rich phase space where the relative orientation of each particle plays a dominant role in the formation of large-scale clusters. The interest in this system is primarily due to the fact that it could give a better understanding of the mechanisms of the formation of polar membranes. A variety of ordered membranelike morphologies is found consisting of single and multiple connected chain configurations. The helicity of these chains can be chosen by simply changing the salt concentration of the solution. Special attention is given to the formation of Bernal spirals. These helices are composed of regular tetrahedra and are known to exhibit nontrivial translational and rotational symmetry.
\end{abstract}

DOI: 10.1103/PhysRevE.92.042309

PACS number(s): 82.70.Dd, 64.75.Yz, 87.16.D-

\section{INTRODUCTION}

The fabrication of arrays of ordered nanoparticles is of interest both for fundamental science related to low-dimensional physics and for applications. Up-to-date lithography is the main approach in generating arrays. This technique is fairly expensive, complicated, and time consuming. An alternative production route to this top-down approach is the bottomup procedure of self-assembly. Monodisperse spheres of submicron size can readily self-assemble into highly ordered and close-packed arrays, so-called colloidal crystals [1]. Most of these initial studies were restricted to isotropic interactions and therefore the possible structures that can be realized were limited. More recently, patchy particles have attracted a large amount of interest. These colloids have an anisotropic interparticle interaction. To realize this anisotropy, there exist different experimental techniques to locally modify the chemical composition and the shape of colloidal particles [2-5]. These local modifications are called patches and depending on the number, size, and chemical properties of each patch these colloids exhibit extraordinary diffusive properties $[6,7]$ or can be used as building blocks for complex structures [8-10]. The substantial research effort in preparing these nanoparticles is driven by their potential applications in nanoelectronics [11], drug delivery [12,13], liquid optics [14,15], and rectification and separation of mixtures $[16,17]$. A special class of patchy particles are Janus particles (JPs). These are colloids that consist of two hemispheres that have different chemical properties, e.g., silica spheres partially covered by gold or platinum. The preparation of Janus nanoparticles is still a major challenge, but different methods have been reported in the literature $[18,19]$.

In the present work we use Janus spheres that are composed of two hemispheres: a hydrophobic side and an electronegatively charged one. The fabrication of these colloidal spheres

\footnotetext{
*mario.sobrinofernandez@uantwerpen.be

†vyacheslav.misko@uantwerpen.be

${ }^{\ddagger}$ francois.peeters@uantwerpen.be
}

was achieved several years ago by depositing a monolayer of octadecanethiol on polystyrene spheres with a thin film of gold on a single hemisphere [20]. When these particles are placed in a solution, the octadecanethiol polymer ionizes and will form an electrical double layer around one hemisphere. Only at a relatively short interparticle distance (with respect to the particle size), the hydrophobic sides will attract each other, while the electrostatic repulsion will repel the particles at a larger length scale (which is defined by the Bjerrum length of the system). In the experiment, the repulsive length scale can be dynamically controlled by adjusting the ionic strength of the solution (which can be chosen by simply changing the salt concentration of the solution) and different assembled structures can be expected. For low ionic strength, the kinetics will be dominated by the electrostatic interaction. Colloids will repel each other regardless of their relative orientation. By increasing the ionic strength of the solution, the screening length of the repulsion can become smaller than the diameter of the colloidal sphere. In this regime the orientation of each colloid becomes of importance. For each value of the ionic strength, there is a value of the angle for which the (total) interparticle force switches from attractive to repulsive [20]. The phase diagram and dynamic properties of Janus particles have been studied previously in great detail [21-23]. How their properties are modified when such particles are confined in a channel-like environment is not yet known. The interest in this system is primarily due to the fact that it could serve as a simple model for membrane formation.

Recently, a new class of artificial amphiphiles was synthesized, known as Janus dendrimeres [24], which were shown to self-assemble in bilayer structures mimicking biological membranes. In turn, Janus particles that combine the amphilicity and colloidal rigidity serve as a good model for Janus dendrimeres. Accordingly, we analyze here various superstructures of Janus spheres that are confined in a narrow channel. This is achieved numerically by placing the Janus spheres in a 3D box and applying a circular symmetric harmonic trap. We explore the interplay between different particle interaction ranges, under the influence of a confinement potential for different particle densities. Using this system, we investigate 
the self-assembly dynamics of Janus particles in a quasione-dimensional channel environment by means of molecular dynamics (MD) simulations using the well-known LAMMPS package [25]. Elongated structures are of particular interest in the development of electronics appliances, such as a singleelectron transistor [26] and high-efficiency photoanodes [27]. More specifically, the system studied can be used to create helical superstructures with an adjustable pitch. For example, the system can be prepared so that the colloids form modified Boerdijk-Coxeter (BC) helices [28,29], an assembly of regular tetrahedra in a twisted fashion with the colloids placed on the vertices. The packing of spheres into a BC helix is closely related to the study of close-packed conformations in biomolecules [30]. Our assembly strategy to obtain these helical structures is the interplay between interactions of different length scales [31]. Competing interactions play a role in protein folding [32] and other polymeric macromolecules. For example, DNA [33] is a polymeric macromolecule that can form a double helix as a result of the interplay between van der Waals and hydrophobic forces. Using Janus colloids in a solution, we show that it is possible to manipulate the helicity of the supermolecule by screening the electrostatic interaction. This approach has been used by adding an electrolyte to the solution (such as $\mathrm{NaCl}$ [3] or polyacrylic acid [34]).

The paper is organized as follows. In Sec. II we discuss the underlying model for Janus particle dynamics, the simulation method, and parameters used. Section III is devoted to the numerical results of the molecular dynamics simulations. We start by determining equilibrium configurations of the system in Sec. III A, followed by Sec. III B discussing the controllability of the helix pitch by changing the interaction length. Special attention is given to the observed Bernard spines with tunable helicity in Sec. III C. Finally, a summary is given in Sec. IV.

\section{MODEL AND SIMULATION TECHNIQUE}

The system of interest consists of hard spheres in a threedimensional geometry. Each spherical colloid is composed

$$
\phi^{I}\left(\boldsymbol{r}_{12}, \hat{\boldsymbol{u}_{1}}, \hat{\boldsymbol{u}_{2}}\right)=\left\{\begin{array}{l}
-\frac{\kappa_{H}}{2}\left(2 R_{H}-r_{12}\right)^{2} \\
Z^{2} \lambda_{B}\left(\frac{\exp (\kappa R)}{1+\kappa R}\right)^{2} \frac{\exp (-\kappa r)}{r}
\end{array}\right.
$$

where $\kappa_{H}$ and $R_{H}$ are elastic parameters that determine the strength and the range of the attraction. The electrostatic repulsion of colloids in solution is governed by a screened Coulomb potential given by the Derjaguin-Landau-VerweyOverbeek (DLVO) [36] theory. Here $Z$ is the effective charge number of the colloidal particles, $\lambda_{B}$ is the Bjerrum length, $\kappa^{-1}$ is the Debeye-Hückel screening length that depends on the solvent, and $\beta^{-1}=k_{B} T$ is the thermal energy of the system at temperature $T$ with $k_{B}$ the Boltzmann constant. Three-dimensional MD simulations are performed with a computational unit cell of 1500 particles confined in a circular symmetric harmonic trap

$$
V(x, y)=\frac{1}{2} K\left(x^{2}+y^{2}\right)
$$

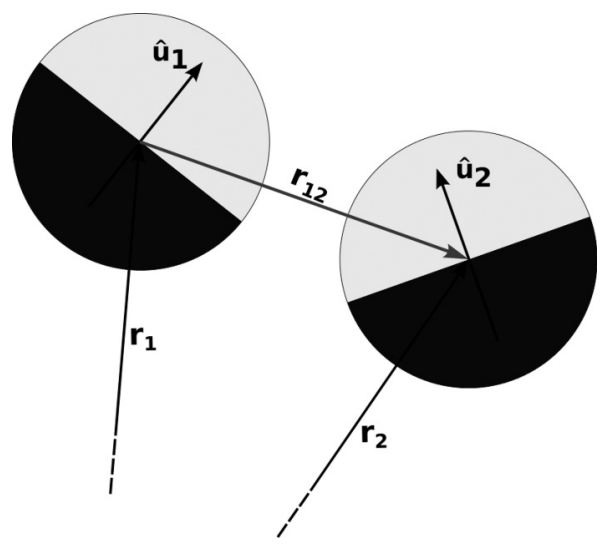

FIG. 1. Configuration of two Janus spheres. The polar axes $\hat{\boldsymbol{u}}_{1}$ and $\hat{\boldsymbol{u}}_{2}$ indicate the center of the electrostatic charge.

of two hemispheres, one being hydrophobic while the other hemisphere has an electrostatic charge. The total pair potential between two spheres placed at $\boldsymbol{r}_{1}$ and $\boldsymbol{r}_{2}$ can be written down in two distinctive parts: an isotropic term representing the hard-sphere repulsion and an angular-dependent factor defined by the patchy structure of the colloidal particles

$$
\phi\left(\boldsymbol{r}_{12}, \hat{\boldsymbol{u}_{1}}, \hat{\boldsymbol{u}_{2}}\right)=\phi^{H C}\left(r_{12}\right)+\phi^{I}\left(\boldsymbol{r}_{12}, \hat{\boldsymbol{u}_{1}}, \hat{\boldsymbol{u}_{2}}\right),
$$

where $\boldsymbol{r}_{12}=\boldsymbol{r}_{1}-\boldsymbol{r}_{2}$ is the separation vector between particles $i$ and $j$ and the $\hat{\boldsymbol{u}}_{i}$ are the polar axes indicating the center of the hydrophobic patch. The hard-core potential as proposed by the Kern-Frenkel model [35] is softened to facilitate the usage of MD simulations, while still having the possibility of tuning the potential so that particles can hardly interpenetrate,

$$
\phi^{H C}\left(r_{12}\right)= \begin{cases}4 \epsilon\left[\left(\frac{2 R}{r_{12}}\right)^{12}-\left(\frac{2 R}{r_{12}}\right)^{6}\right], & r_{12}<2 R \\ 0, & r_{12}>2 R,\end{cases}
$$

where $R$ is the radius of the Janus sphere. The anisotropic contribution $\phi^{I}$ depends on the relative orientation between two particles (see Fig. 1)

and periodic boundary conditions are applied in the perpendicular direction, forcing the colloids in a quasi-one-dimensional state. In this work the strength of the potential will not be altered as its sole purpose is to localize particles in the center of the trap. The size of the rectangular unit cells measures $50 R$ in the $x$ or $y$ direction and $2000 R$ in the $z$ direction. We study the motion of the Janus particles by using a Verlet algorithm. The Lennard-Jones potential in Eq. (2) is shifted [37] so that both the potential and the force continuously approach zero at the cutoff $r_{12}=2 R$. The angular-dependent part of the pair potential is transformed into a $C^{2}$ function by performing local $r^{4}$ smoothening near the boundaries of the patches. The temperature of the solution is fixed using a Nosé-Hoover thermostat. Annealing is done starting from $300 \mathrm{~K}$ to $0 \mathrm{~K}$ to find the minimum energy structure. Parameters are chosen 
(a)
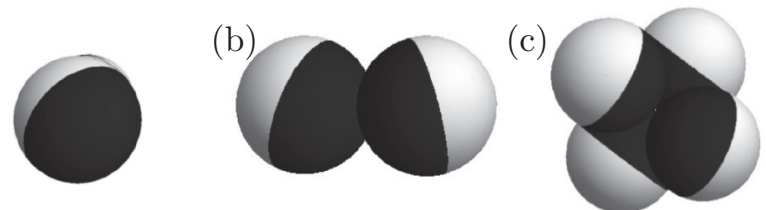

(d)

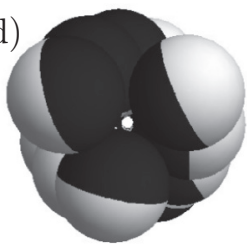

e)

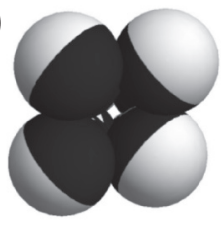

(f)

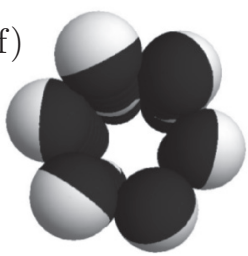

FIG. 2. Basal area of different elongated superstructures at increasing packing fraction for $\kappa R=0.95$ : (a) single chain $(\eta \leqslant 2 \times$ $\left.10^{-3}\right)$, (b) double chain $\left(2 \times 10^{-3} \leqslant \eta \leqslant 4 \times 10^{-3}\right)$, (c) rotated dimer $\left(4 \times 10^{-3} \leqslant \eta \leqslant 4.5 \times 10^{-3}\right)$, (d) Bernal spiral $\left(4.5 \times 10^{-3} \leqslant \eta \leqslant\right.$ $\left.6.5 \times 10^{-3}\right)$, (e) rectangular chain $\left(6.5 \times 10^{-3} \leqslant \eta \leqslant 8 \times 10^{-3}\right)$, and (f) a chainlike structure with a pentagon at its base $\left(\eta \geqslant 8 \times 10^{-3}\right)$.

such that particles can hardly interpenetrate. If the solvent has a weak ionic strength, the electrostatic repulsion is so strong that particles repel each other regardless of their relative orientation. This effect weakens strongly as soon as the DebeyeHückel screening length $\kappa^{-1}$ becomes smaller than the particle size. At this point more particle orientations become energetically stable and more structures can be obtained. Parameters are chosen to be similar to those used in Ref. [20], unless stated otherwise: $R=1 \mu \mathrm{m}, D=0.2 T \mu \mathrm{m}^{2} / \mathrm{s}, \epsilon=2 \mathrm{~N} / \mu \mathrm{m}$, $R_{H}=1.15 \mu \mathrm{m}, \kappa_{H}=0.1 \mathrm{~N} / \mu \mathrm{m}, Z=0.32 e, \lambda_{B}=2 \mu \mathrm{m}$, $\kappa=1.4 \mu \mathrm{m}^{-1}, K=0.1 \mathrm{~N} / \mathrm{m}$, and $\Delta t=0.005 \mathrm{~s}$.

\section{NUMERICAL RESULTS}

\section{A. Morphologies}

By varying the concentration of particles inside the channel, we find a series of different morphologies, which we will characterize here by the geometric shape of the basal area. The complete morphology diagram is remarkably rich: For each geometric shape of the basal area, different superstructures exist with tunable helical properties, which we will address in the following section. In this part, however, we focus only on periodic superstructures without particular helical properties. Assembling superstructures with different geometries can be done merely by altering the packing fraction. The emergence of chirality, on the other hand, is the result of a competition between interactions with different length scales. This has been shown previously in other colloidal systems such as asymmetric dumbbells [38,39] and disklike ellipsoids [40]. Here we show the same mechanism for spherical particles, where the asymmetric length scales are a result of patch interaction rather than using asymmetric colloids. In Fig. 2 the different superstructures are shown for increasing packing fraction $\eta=4 / 3 N_{\text {part }} \pi R^{3} / L_{x} L_{y} L_{z}$, where $N_{\text {part }}$ is the number of particles of size $4 / 3 \pi R^{3}$ in a simulation box lengths $L$.

For a packing fraction $\eta \leqslant 2 \times 10^{-3}$ all the colloids trapped in the parabolic confinement can occupy the center of the trap [Fig. 2(a)], forming a single chain in the vertex of the trap. For

(a) 10000000000000000000000000

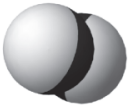

(b)
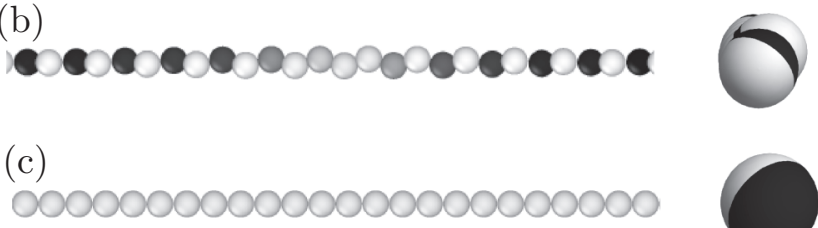

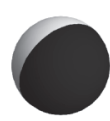

FIG. 3. Different helical structures for varying the electrostatic interaction range for $\eta=10^{-3}$ : (a) planar zigzag chain for $\kappa R=0.05$, (b) one full rotation of a helical chain for $\kappa R=0.35$, and (c) straight chain for $\kappa R=0.95$. The particles in the chain are colored by their inclination in the $Z$ direction. On the right-hand side, the basal area of the chains is shown, in accord with Fig. 2.

$\eta \geqslant 2 \times 10^{-3}$ it becomes unattainable for all the colloids to position themselves in the center and a double chain is formed [Fig. 2(b)]. The apolar patch of the Janus sphere is attracted to the interior of the structure and the polar charged hemisphere is presented to the outside of the structure. The formation of two antiparallel chains is favorable both for the masked hydrophobic patch and for the charged patch, which attains a low number of neighboring charges. For $\eta>4 \times 10^{-3}$ also this density restricted configuration reaches its limit. Before the superstructure switches to a triangular morphology, there exists an intermediate structure where each sequential dimer is rotated by $90^{\circ}$, reducing the length of the total complex [Fig. 2(c)]. This state is stable in a small region of densities up to $\eta>4.5 \times 10^{-3}$. Here we find a helical assembly with a triangular base [Fig. 2(d)]. Helical assemblies will be discussed in the following sections, but it is worth mentioning here that no straight complexes with a triangular base are found. We will discuss this tetrahelix with great detail in Sec. III C. Following this, we again find a straight complex with a square base in the range $6.5 \times 10^{-3} \leqslant \eta \leqslant 8 \times 10^{-3}$ [Fig. 2(e)], above which the system reorders itself to form a pentagon [Fig. 2(f)]. At even higher packing fractions $\left(\geqslant 1.2 \times 10^{-2}\right)$, the spacing in between the base is large enough to fit a particle at its center and no periodic elongated structures are observed.

(a)

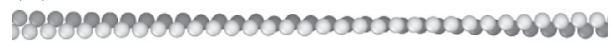

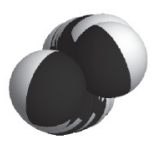

(b) 8080800808080808080880808080800800

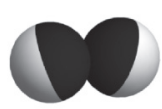

FIG. 4. Helical superstructure consisting of (a) two intertwined spirals ( $\eta=3 \times 10^{-3}$ ) (inset showing one helix turn) for $\kappa R=0.25$ and (b) the corresponding straight complex for $\kappa R=0.95$. 
(a)

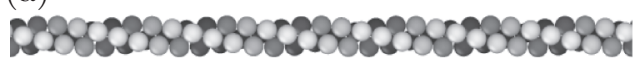

(b)

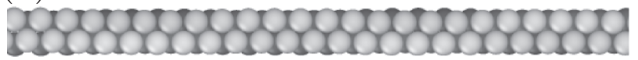

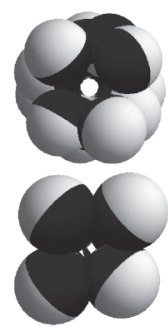

FIG. 5. Helical superstructure at $\eta=7 \times 10^{-3}$ consisting of (a) four intertwined spirals for $\kappa R=0.15$ and (b) the corresponding straight complex for $\kappa R=0.95$.

\section{B. Tunable pitch}

By altering the range of the electrostatic repulsion, we find that the colloidal building blocks can form superstructures with a tunable pitch. This effect has been observed for the different helical structures that were produced in our simulations. In Fig. 3 three different morphologies are given that arise when varying solely the value of $\kappa R$. For a low value of $\kappa R$ (which coincides with a long-range interaction), the resulting superstructure is a planar zigzag chain. When we decrease the length of the electrostatic repulsion, we find the same zigzag structure, twisted in a helix. Reducing the interaction range even further, we find a straight linear chain of colloids, consistent with what would be expected as a solution of hard spheres in a confinement potential without any additional interparticle interactions. By simply altering the interaction range of the repulsive component, the morphology can be altered between linear, planar, or helical.

This mechanism of unfolding a helix when decreasing the interaction range can also be seen for more complex structures. In Figs. 4 and 5 we observe the same mechanism at higher packing fractions for a helix that contains two $\left(\eta=3 \times 10^{-3}\right)$ and four $\left(\eta=7 \times 10^{-3}\right)$ intertwined strands. When gradually reducing the range of the repulsive interaction between the charged hemisphere of the JPs, the helix unravels and we are left with two or four straight chains of JPs with opposite orientation towards the center of the complex.

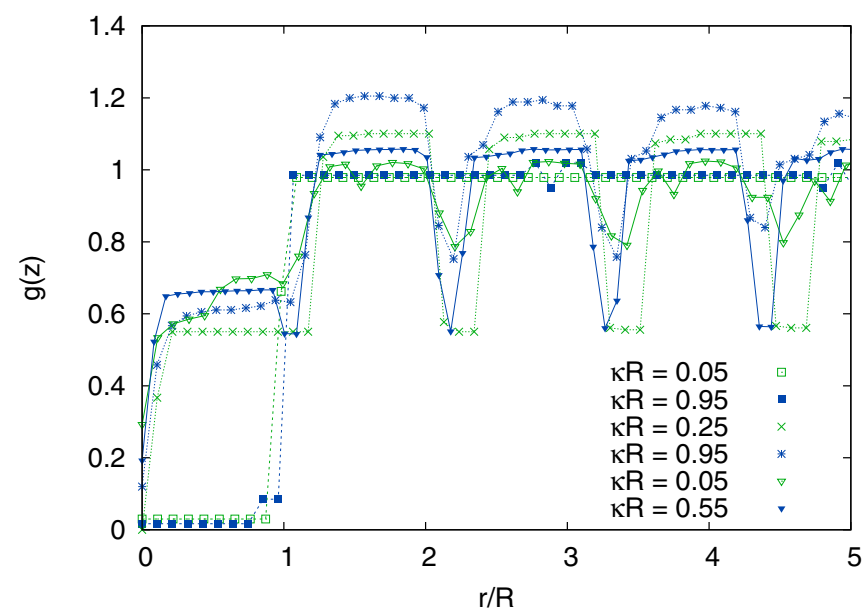

FIG. 6. (Color online) Tunable pitch of superstructures using $g(z)$ as given in Eq. (5), for a single helix [squares and dotted line (see Fig. 3)], double helix [crosses and dashed line (see Fig. 4)], and quadruple helix [triangles and solid line (see Fig. 5)].

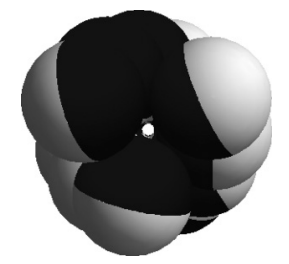

FIG. 7. Top view of a Boerdijk-Coxeter helix, which consists of three intertwined chains, where the position of each particle can be constructed from Eq. (6).

In Fig. 6 the pair correlation function is given for the previously discussed helical assemblies. The pair correlation function for colloids in a channel will be defined as Ref. [41]

$$
g(z)=\frac{1}{\rho}\left\langle\sum_{i} \sum_{j} \delta\left(z_{i}\right) \delta\left(z_{j}-z\right)\right\rangle,
$$

where $\rho$ is the average number of particles in the direction of the channel. This function does not take into account displacements in the $x$ or $y$ direction. As the system is quasi-one-dimensional, using a 2D or 3D correlation function would result in general long-range behavior. Only typical oscillations that are due to the periodicity of the structure are observed. Long-range correlations corresponding to the helicity of the structure are diminished by averaging over all particles and are not visible in Fig. 6.

\section{Boerdijk-Coxeter helix}

A BC helix is a helical assembly of regular tetrahedra, arranged so that the edges of the complex form three intertwined helices with a nontrivial translational or rotational symmetry.

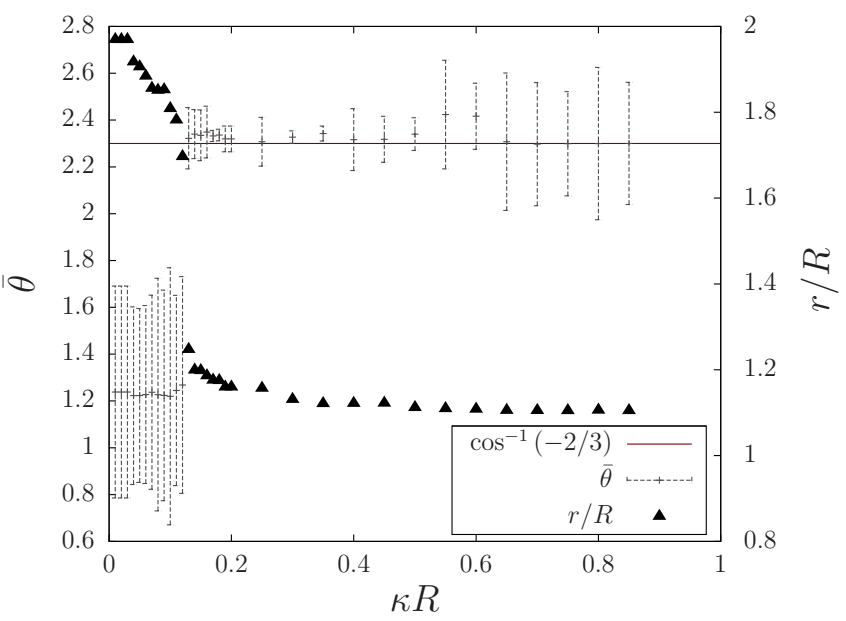

FIG. 8. (Color online) The BC helices are characterized by the sequence given by Eq. (6). The triangles are the value of $r=$ $3 \sqrt{3} / 10 L_{E}$ and correspond to the edge length of the tetrahedra, while $\theta$ is the angle between sequential particles in the helix. For $\kappa R \simeq 0.12$ a sharp transition is observed between a single helix and a Boerdijk-Coxeter triple helix, which is also apparent in the value for the radius $r$ of the helix. For larger values of $\kappa R$ the BC state becomes less dominant in the system, which can be seen by the large variance of $\theta$. 


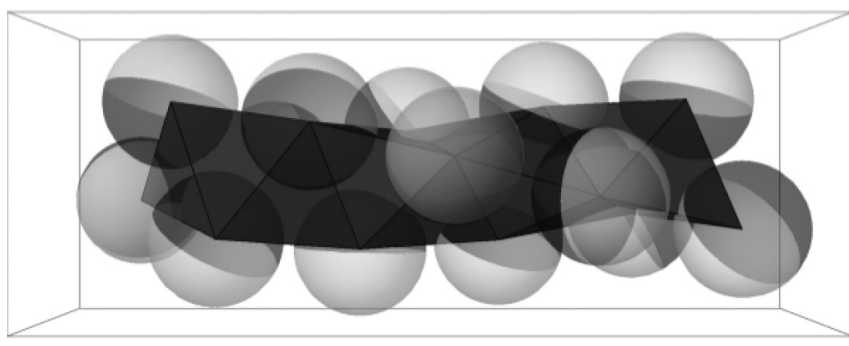

FIG. 9. Illustration of a $\mathrm{BC}$ helix found in simulations alongside a reconstruction of the underlying tetrahelix structure as described by Eq. (6).

Although one would expect to find the thermodynamically favored 3(0,1,1) helix [42] (a helical structure with three particles at its base arranged in a triangular lattice), these structures have been found experimentally in solutions of Janus particles at high particle concentration [43]. Their appearance has been accredited to being kinetically more favorable: During the initial formation of the helix, the base building block of a BC helix (which is a capped trigonal bipyramidal isomer) is preferred over the octahedrol isomer that forms $3(0,1,1)$ helices. Once formed, the relaxation of a BC helix into a $3(0,1,1)$ helix would require a collective transition, in which for each group of three particles at least one bound must be broken. Using the distilled framework of molecular dynamics simulations, we find that these metastable $\mathrm{BC}$ helices indeed are found in a wide range of $\kappa R$ values $(0.12 \leqslant \kappa R \leqslant 1)$, while a $3(0,1,1)$ helix is never observed.

The coordinates for the vertices of these tetrahedral structures are given by the sequence [44]

$$
s_{n}=[r \cos (n \theta), \pm r \sin (n \theta), n h],
$$

where $\quad r=3 \sqrt{3} / 10 L_{E}, \quad \theta=\arccos (-2 / 3), \quad$ and $\quad h=$ $L_{E} / \sqrt{10}$, with $L_{E}$ the edge length of the tetrahedra. The \pm sign in front of the second term determines the chirality of the helix. Due to the irrational nature of $\theta$, it is clear that the $\mathrm{BC}$ helix will have an aperiodic nature. A top-down view of the structure can be seen in Fig. 7 .

In Fig. 8 we show the transition between a large single spiral and a close-packed Boerdijk-Coxeter helix. This is accomplished by varying the screening length (which can be easily done in an experiment by changing the ion concentration of the solution) over a broad range of $\kappa R$ values. For small values of $\kappa R$ the repulsion between neighboring particles is strong enough to overcome the strength of the confinement potential, regardless of their relative orientation, and no bound state is present. For a critical value of $\kappa R$, the interaction between particles will be determined by their orientation towards one another and the structure transforms to a triple helix. We find that the angle $\theta$ as introduced in Eq. (6) becomes well defined for the entire structure and more importantly equal to $\arccos (-2 / 3)$, in agreement with the definition of a $\mathrm{BC}$ helix. As the value of $\kappa R$ is increased further, we find that the spread on the mean value of $\theta$ increases. In this regime the repulsion between the charged hemispheres is weak enough to allow more stochastic variations on the orientation of the JPs without decomposing the structure. In Fig. 9 the underlying tetrahelix structure is shown for $\kappa R=0.18$. We find excellent agreement between the tetrahedra as described by Eq. (6) and the helical packing of Janus spheres.

\section{CONCLUSION}

Using molecular dynamics simulations, we studied a system of Janus particles weakly confined in a three-dimensional channel. Each particle consists of a charged hemisphere and a hydrophobic one. The electrostatic repulsion between colloids in solution takes the form of a screened-Coulomb potential given by the DLVO theory. The different morphologies were discussed for a wide range of packing fractions in the channel. The basic Janus pair dynamics of shielding the hydrophobic patch from the liquid on the one hand and maximizing the distance between charged hemispheres gives rise to a rich variety of morphologies, which are identified according to the arrangement of the particles in a single base. A striking property of these chains is that their helicity can be tuned by altering the interaction range of the electrostatic repulsion, which can be easily achieved experimentally by altering the ionic strength of the solution. We showed the formation of helices for single, double, triple, and quadruple chains. Finally, the formation of Boerdijk-Coxeter helices was discussed. Our results reinforce the importance of kinetic selection in the formation of $\mathrm{BC}$ helices, as first observed in an experiment by Chen et al. [43]. Whereas the experimental setup consisted of a large network of wormlike structures, we found that $\mathrm{BC}$ helices indeed are found in a wide range of $\kappa R(0.12 \leqslant \kappa R \leqslant 1)$ in an uncluttered system. Furthermore, we showed that the proclaimed $\mathrm{BC}$ helix indeed has an aperiodic sequence and is the most favored structure in a wide range of electrostatic screening lengths.

\section{ACKNOWLEDGMENTS}

This work was supported by the Fund for Scientific Research Flanders (FWO) and by the "Odysseus" program of the Flemish government and FWO.
[1] R. J. Macfarlane, B. Lee, M. R. Jones, N. Harris, G. C. Schatz, and C. A. Mirkin, Science 334, 204 (2011).

[2] A. B. Pawar and I. Kretzschmar, Langmuir 24, 355 (2008).

[3] S. Sacanna, L. Rossi, and D. J. Pine, J. Am. Chem. Soc. 134, 6112 (2012).

[4] S. Sacanna and D. J. Pine, Curr. Opin. Colloid Interface Sci. 16, 96 (2011).
[5] K. P. Yuet, D. K. Hwang, R. Haghgooie, and P. S. Doyle, Langmuir 26, 4281 (2010).

[6] G. Volpe, I. Buttinoni, D. Vogt, H.-J. Kummerer, and C. Bechinger, Soft Matter 7, 8810 (2011).

[7] L. Baraban, R. Streubel, D. Makarov, L. Han, D. Karnaushenko, O. G. Schmidt, and G. Cuniberti, ACS Nano 7, 1360 (2013).

[8] M. J. Solomon, Curr. Opin. Colloid Interface Sci. 16, 158 (2011). 
[9] S. C. Glotzer and M. J. Solomon, Nat. Mater. 6, 557 (2007).

[10] G. Doppelbauer, E. Bianchi, and G. Kahl, J. Phys.: Condens. Matter 22, 104105 (2010).

[11] Y. Xiang, S. Lu, and S. P. Jiang, Chem. Soc. Rev. 41, 7291 (2012).

[12] J. Li, C. Fan, H. Pei, J. Shi, and Q. Huang, Adv. Mater. 25, 4386 (2013).

[13] M. J. Campolongo, S. J. Tan, J. Xu, and D. Luo, Adv. Drug Delivery Rev. 62, 606 (2010).

[14] T. Nisisako, T. Torii, T. Takahashi, and Y. Takizawa, Adv. Mater. 18, 1152 (2006).

[15] M. A. Bucaro, P. R. Kolodner, J. A. Taylor, A. Sidorenko, J. Aizenberg, and T. N. Krupenkin, Langmuir 25, 3876 (2009).

[16] W. Yang, V. R. Misko, K. Nelissen, M. Kong, and F. M. Peeters, Soft Matter 8, 5175 (2012).

[17] P. K. Ghosh, V. R. Misko, F. Marchesoni, and F. Nori, Phys. Rev. Lett. 110, 268301 (2013).

[18] A. Walther and A. H. E. Müller, Soft Matter 4, 663 (2008).

[19] M. Lattuada and T. A. Hatton, Nano Today 6, 286 (2011).

[20] L. Hong, A. Cacciuto, E. Luijten, and S. Granick, Langmuir 24, 621 (2008).

[21] F. Sciortino, A. Giacometti, and G. Pastore, Phys. Rev. Lett. 103, 237801 (2009).

[22] G. Rosenthal, K. E. Gubbins, and S. H. L. Klapp, J. Chem. Phys. 136, 174901 (2012).

[23] M. S. Fernández, V. R. Misko, and F. M. Peeters, Phys. Rev. E 89, 022306 (2014).

[24] B. M. Rosen, C. J. Wilson, D. A. Wilson, M. Peterca, M. R. Imam, and V. Percec, Chem. Rev. 109, 6275 (2009).

[25] S. Plimpton, J. Comput. Phys 117, 1 (1995).

[26] R. S. Gill, R. F. Saraf, and S. Kundu, ACS Appl. Mater. Interfaces 5, 9949 (2013).
[27] S. H. Ko, D. Lee, H. W. Kang, K. H. Nam, J. Y. Yeo, S. J. Hong, C. P. Grigoropoulos, and H. J. Sung, Nano Lett. 11, 666 (2011).

[28] A. Boerdijk, Philips Res. Rep. 7, 303 (1952).

[29] H. S. M. Coxeter, Regular Complex Polytopes (Cambridge University Press, London, 1974).

[30] J. F. Sadoc and N. Rivier, Eur. Phys. J. B 12, 309 (1999).

[31] S. N. Fejer, D. Chakrabarti, H. Kusumaatmaja, and D. J. Wales, Nanoscale 6, 9448 (2014).

[32] E. I. Shakhnovich, Curr. Opin. Struct. Biol. 7, 29 (1997).

[33] J. D. Watson and F. H. C. Crick, Nature (London) 171, 737 (1953).

[34] H. Jans, K. Jans, L. Lagae, G. Borghs, G. Maes, and Q. Huo, Nanotechnology 21, 455702 (2010).

[35] N. Kern and D. Frenkel, J. Chem. Phys. 118, 9882 (2003).

[36] B. V. Derjaguin and L. Landau, Acta Phys. Chim. URSS 14, 633 (1941).

[37] S. Toxvaerd and J. C. Dyre, J. Chem. Phys. 134, 081102 (2011).

[38] D. Chakrabarti, S. N. Fejer, and D. J. Wales, Proc. Natl. Acad. Sci. USA 106, 20164 (2009).

[39] D. Zerrouki, J. Baudry, D. Pine, P. Chaikin, and J. Bibette, Nature (London) 455, 380 (2008).

[40] S. N. Fejer and D. J. Wales, Phys. Rev. Lett. 99, 086106 (2007).

[41] C.-Y. Chou, B. Payandeh, and M. Robert, Phys. Rev. E 73, 041409 (2006).

[42] R. O. Erickson, Science 181, 705 (1973).

[43] Q. Chen, J. K. Whitmer, S. Jiang, S. C. Bae, E. Luijten, and S. Granick, Science 331, 199 (2011).

[44] R. W. Gray, Tetrahelix data, http://www.rwgrayprojects.com/ rbfnotes/helix/helix01.html (2013). 